\title{
A Calibration Method for Structural Models of Credit Risk with Reporting Bias
}

\author{
Agostino Capponi
}

\begin{abstract}
We propose a novel calibration methodology based on the maximum likelihood estimator to recover the parameters of a structural model of credit risk which accounts for potential reporting bias. Such bias is introduced by the managers and it is unobserved by outsider investors which can only estimate it. The calibration is performed using a combination of balance sheet, financial indicators and market prices of equities. We apply the calibration algorithm to Tyco, a real case of reporting bias in the United States history. We show that the calibrated model is able to predict the market stock price with a high degree of accuracy.
\end{abstract}

\section{INTRODUCTION}

The valuation of financial assets, as well as hedging and trading decisions, are often based on signals. Those signals may represent a faithful representation of the fundamentals driving the value of the assets and the performance of financial strategies, or they may contain noise which needs to be filtered out in order to obtain consistent market prices. For some markets, such as interest rate and foreign exchange, the perfect information assumption may be a reasonable approximation of reality. However, this is not the case for many others, the most obvious example being the market of corporate securities. In this market, information asymmetries may severely affect the design of the financial products as well as their prices. The problem of transparency is extremely important for credit risk models, particularly for the so called structural approach. In these models, the values of debt and equity securities are recovered from a representation of the balance sheet information, therefore the quality of accounting information plays a key role. The literature on structural models goes back to Merton's work [11], where the firm defaults if, at the time of servicing the debt, its assets are below its outstanding debt. Merton's model assumes that the firm's dynamic is regulated by a diffusion process and that the value of the firm is absolutely transparent to outsiders. Under this framework, Duan [6] [7] proposed a methodology that uses the market prices of stocks to compute maximum likelihood parameter estimates for the drift and the volatility of the asset value process.

A recent stream of literature has tried to address the issue of accounting transparency. The seminal paper goes back to [8], who proposes a structural model with endogenous default threshold, but in which the market only observes noisy or delayed accounting reports from which investors have to

Agostino Capponi is with the Division of Engineering and Applied Sciences, California Institute of Technology, Pasadena, CA (email: acapponi@caltech.edu).

This work was supported by a SISL fellowship granted by the Information and Sciences Laboratory at Caltech. 2050375 draw inference of the true asset value of the firm. Such approach has been complemented and extended by several other contributions. Yu [12] proved that accounting noise is actually priced in the market by showing empirically that a risk premium is charged to the credit spreads of firms that adopt less transparency. The authors in [5] generalize the model by incorporating accounting noise in continuous time in a model that precludes arbitrage from both insiders and outsiders. In [4] and [9], the authors present a framework in which the market is assumed to only partially observe, and possibly with a lag, relevant information concerning the state of the firm.

We present a novel calibration methodology for a simple version of the credit model with reporting bias developed by the authors in [3]. Such methodology uses a set of balance sheet indicators and stock market prices to recover maximum likelihood parameter estimates. The proposed method generalizes Duan's approach and reduces to it in case when no reporting bias is accounted for in the model. We apply this calibration method to Tyco, a well known case of reporting bias in the United States history. We show that, after accounting for the calibrated amount of reporting bias, we can predict the market stock price of equity with a high degree of accuracy.

The rest of the paper is organized as follows. Section II defines a simple version of the credit risk model with reporting bias developed in [3] and presents the pricing formulas for equity and bonds. Section III contains the main contribution of the paper which consists of a novel calibration methodology. Section IV uses the developed method to estimate the expected amount of reporting bias introduced by Tyco executives, and then predict the market stock price of equities. Section V concludes the paper. More results and technical proofs are provided in the Appendix.

\section{The CREDit Model With REPORTING BIAS}

\section{A. The Mathematical Model}

We assume that there is a price process $V_{t}$ that represents the true value of the firm's asset. It generates a filtration denoted by $\mathcal{F}_{t}$ and evolves according to the following dynamics

$$
d V_{t}=\mu V_{t} d t+\sigma V_{t} d W_{t}
$$

where $\mu$ and $\sigma$ are deterministic parameters representing respectively the drift and volatility of the assets. There is a process $y_{t}$ which represents the log-asset value of the firm which is revealed to outsider investors. The actual asset value may be deliberately biased by the insider managers and executives to make the firm look more profitable than it is. 


\begin{tabular}{|c|c|c|c|}
\hline & Assets & Bonds & Equity \\
\hline \hline No default & $V_{T} \geq K$ & $K$ & $V_{T}-K$ \\
\hline Default & $V_{T}<K$ & $V_{T}$ & 0 \\
\hline
\end{tabular}

TABLE I

PAYOFF AT MATURITY FOR EQUITY AND BOND HOLDERS

Such released log-asset value $y_{t}$ is defined by the following equation

$$
y_{t}=\log \left(V_{t}\right)+h(t)+\nu u_{t}
$$

where $u_{t}$ is a gaussian white noise sequence, here assumed to be uncorrelated with $W_{t}, \nu$ is a scalar parameter and $h(t)$ denotes the expected amount of reporting bias occurred at time $t$. We denote by $Y^{t}$ the filtration generated by the process $\left\{y_{s}\right\}$ up to time $t$.

We work under the same hypotheses of Merton, i.e. we assume that the firm is financed by equity and a zero coupon bond with face value $K$ and maturity date $T$. The firm's contractual obligation is to repay the amount $K$ to the bond investors at time $T$. Debt covenants grant bond investors absolute priority: if the firm cannot fulfill its payment obligation, then bond holders will immediately take over the firm. The payoff to equity and bond holders are summarized in Table I. Let us denote by

$$
\tau=T-t
$$

the time to maturity for the bond. It has been shown in [3] that, under the above setting, the pricing formulas for bond and equity are respectively given by

$$
B(t, T)=e^{-r \tau}\left(K-\mathbb{E}^{\mathbb{P}^{*}}\left[\left(K-V_{T}\right)^{+} \mid Y^{t}\right]\right)
$$

and

$$
E(t, T)=e^{-r \tau} \mathbb{E}^{\mathbb{P}^{*}}\left[\left(V_{T}-K\right)^{+} \mid Y^{t}\right]
$$

We next discuss the two main assumptions needed to make the model well defined in an arbitrage free pricing setting, thus identifying the pricing measure $\mathbb{P}^{*}$. They are:

- Insider Regulation. The true asset value $V_{t}$ cannot be traded. Such assumption prevents people endowed with superior information to intervene in market trading. Such assumption has been made in many works, see [8], for example.

- No Outsider arbitrage. The innovation process $\mathbb{E}\left[V_{t} \mid Y^{t}\right]$ is equal to the sum of equity and bond value, namely

$$
\mathbb{E}^{\mathbb{P}^{*}}\left[V_{t} \mid Y^{t}\right]=E(t, T)+B(t, T)
$$

where the pricing measure $\mathbb{P}^{*}$ is obtained replacing the drift $\mu$ with the risk free interest rate $r$. A proof of this fact is reported in Appendix I. Therefore, being equity and bonds tradeable assets, we need to prevent outsiders from using the information gain obtained from their model along with the set of released observations to make arbitrage. This is equivalent to impose the requirement that $\mathbb{E}\left[V_{t} \mid Y^{t}\right]$ is a martingale with respect to the outsider filtration $Y^{t}$. We verify that this is the case under the same pricing measure $\mathbb{P}^{*}$ in Appendix II.

- No insider trading. The insiders are prohibited to trade $\mathbb{E}^{\mathbb{P}^{*}}\left[V_{t} \mid Y^{t}\right]$, thus the innovation process does not have to be a martingale with respect to the insider filtration $\mathcal{F}_{t}$.

\section{B. Pricing Formulas}

We report the explicit expressions for bond and equity price under the credit risk framework described in subsection II-A. Let us denote by

$$
x_{t}=\log \left(V_{t}\right)
$$

the actual $\log$ asset value of the firm. Let us define the conditional mean and conditional variance

$$
\hat{x}_{t \mid t}=\mathbb{E}\left[x_{t} \mid Y^{t}\right], \quad \hat{\sigma}_{t \mid t}^{2}=\mathbb{E}\left[\left(x_{t}-\hat{x}_{t \mid t}\right)^{2} \mid Y^{t}\right]
$$

and the predicted variance

$$
\hat{\sigma}_{T \mid t}=\sqrt{\hat{\sigma}_{t \mid t}^{2}+\sigma^{2}(T-t)}
$$

Moreover, let us define

$$
\begin{aligned}
& d_{1}(x, K, \tau, v)=\frac{x-\log (K)+\left(r+0.5 \sigma^{2}\right) \tau}{v} \\
& d_{2}(x, K, \tau, v)=d_{1}(x, K, \tau)-v
\end{aligned}
$$

It can be proven, see [3], that the prices of bond and equity securities under the above framework are respectively given by

$$
\begin{aligned}
B(t, T)= & K e^{-r \tau}\left(1-N\left(-d_{2}\left(\hat{x}_{t \mid t}, K, \tau, \hat{\sigma}_{T \mid t}\right)\right)\right)- \\
& e^{\hat{x}_{t \mid t}+0.5 \hat{\sigma}_{t \mid t}^{2}} N\left(-d_{1}\left(\hat{x}_{t \mid t}, K, \tau, \hat{\sigma}_{T \mid t}\right)\right)
\end{aligned}
$$

and

$$
\begin{aligned}
E(t, T)= & e^{\hat{x}_{t \mid t}+0.5 \hat{\sigma}_{t \mid t}^{2}} N\left(d_{1}\left(\hat{x}_{t \mid t}, K, \tau, \hat{\sigma}_{T \mid t}\right)\right)- \\
& K e^{-r \tau} N\left(d_{2}\left(\hat{x}_{t \mid t}, K, \tau, \hat{\sigma}_{T \mid t}\right)\right)
\end{aligned}
$$

where $N(x)$ denotes the standard univariate gaussian distribution function.

\section{THE CALIBRATION METHOD}

We develop a calibration algorithm which allows to estimate the expected amount of reporting bias $h(t)$, the drift $\mu$, the volatility $\sigma$, and the standard deviation $\nu$ of the noise in the reported asset value $y_{t}$.

\section{A. The discrete framework}

We discretize the system given by Eq. (1) and Eq. (2) and work on a logarithmic scale, thus having

$$
\begin{aligned}
x_{k} & =x_{k-1}+\left(\mu-0.5 \sigma^{2}\right) \Delta_{k}+\sigma\left(W_{k}-W_{k-1}\right) \\
y_{k} & =x_{k}+h(k)+\nu u_{k}
\end{aligned}
$$

Let us denote by $\Delta_{k}=t_{k}-t_{k-1}$ the time between consecutive observations. The filter density is Gaussian with 
mean $\hat{x}_{k \mid k}$ and variance $\sigma_{k \mid k}^{2}$ given by the Kalman filter [10] as

$$
\left(\hat{x}_{k \mid k}, \hat{\sigma}_{k \mid k}^{2}\right)=\mathbf{K F}\left(\hat{x}_{k-1 \mid k-1}, \hat{\sigma}_{k-1 \mid k-1}^{2}, y_{k}, h(k), \nu, \mu, \sigma\right)
$$

where the recursive procedure is given by the following system of equations.

$$
\begin{aligned}
\hat{x}_{k \mid k-1} & =\hat{x}_{k-1 \mid k-1}+\left(\mu-0.5 \sigma^{2}\right) \Delta_{k} \\
\hat{\sigma}_{k \mid k-1}^{2} & =\hat{\sigma}_{k-1 \mid k-1}^{2}+\sigma^{2} \Delta_{k} \\
\hat{y}_{k \mid k-1} & =\hat{x}_{k \mid k-1}+h(k) \\
\hat{\nu}_{k \mid k-1}^{2} & =\hat{\sigma}_{k \mid k-1}^{2}+\nu^{2} \\
G_{k} & =\frac{\hat{\sigma}_{k \mid k-1}^{2}}{\hat{\sigma}_{k \mid k-1}^{2}+\nu^{2}} \\
\hat{x}_{k \mid k} & =\hat{x}_{k \mid k-1}+G_{k} \cdot\left(y_{k}-\hat{x}_{k \mid k-1}\right) \\
\hat{\sigma}_{k \mid k}^{2} & =\left(1-G_{k}\right) \cdot \hat{\sigma}_{k \mid k-1}^{2}
\end{aligned}
$$

\section{B. The expected cost of reporting bias}

We may express the expected amount of reporting bias $h(k)$ as

$$
h(k)=P_{m}(k) E[m(k) \mid\{m(k)>0\}]
$$

where the random variable $m(k)$ denotes the amount of reporting bias occurring at time $t_{k}$, and $P_{m}(k)$ denotes the probability that a reporting bias larger than zero was introduced at time $t_{k}$. We employ a methodology developed in [13] to estimate the probability $P_{m}(k)$ of reporting bias at time $t_{k}$. We do not reproduce their work here, but briefly recall it. Such methodology recovers the amount of reporting bias from a set of financial and balance sheet indicators using a bivariate-probit model as follows. Let $F_{i}^{*}$ denote the potential of firm $i$ to bias reporting, and $D_{i}^{*}$ the potential of firm $i$ to get discovered if the reporting is biased event occurs. Then

$$
\begin{aligned}
F_{i} & =\mathbf{z}_{F, i} \boldsymbol{\beta}_{F}+u_{i} \\
D_{i} & =\mathbf{z}_{D, i} \boldsymbol{\beta}_{D}+v_{i}
\end{aligned}
$$

where $\mathbf{z}_{F, i}$ is a row vector whose components are factors explaining the potential of firm $i$ to bias reporting, and $\mathbf{z}_{D, i}$ is a row vector whose components are factors explaining the potential of firm $i$ to get detected if reporting is biased, and $u_{i}, v_{i}$ are Gaussian random variables with correlation coefficient $\rho$. Using a sample of firms belonging to a heterogeneous number of sectors (see [13] for details), they recover the maximum likelihood estimates $\hat{\boldsymbol{\beta}}_{F}$ and $\hat{\boldsymbol{\beta}}_{D}$ of their model parameters. We take the model coefficient estimates as computed by their procedure, and report their values in Table II. We then feed the model in Eq. (23) with the predictors computed using balance sheet data of our specific firm at a time $t_{k}$, let them be $\mathbf{z}_{F}(k)$ and $\mathbf{z}_{D}(k)$, and compute the probability $P_{m}(k)$ as

$$
P_{m}(k)=N\left(\hat{\boldsymbol{\beta}}_{F} \mathbf{z}_{F}(k)\right)
$$

\section{The transformed maximum likelihood estimator}

We next describe a novel maximum likelihood estimator procedure which recovers the system parameters from a time series of market prices of equity. We imply the reported logasset values $y_{k}$ from the market stock prices. This differs from Duan [6] [7], who implies the exact asset values $V_{k}$ from stock prices. Let us first assume that a sample of released observations $\left\{y_{1}, y_{2}, \ldots, y_{n}\right\}$ was observed on the market at discrete dates $\left\{t_{1}, t_{2}, \ldots, t_{n}\right\}$. The log-likelihood function associated to the system of Eq. (13) is given by:

$$
\begin{aligned}
L L\left(y_{1}, y_{2}, \ldots, y_{n}\right)= & -\frac{n}{2} \log (2 \pi)-\sum_{i=1}^{n} \log \left(\hat{\nu}_{i \mid i-1}\right) \\
& -\sum_{i=1}^{n} \frac{\left(y_{i}-\hat{y}_{i \mid i-1}\right)^{2}}{2 \hat{\nu}_{i \mid i-1}^{2}}
\end{aligned}
$$

where $\hat{y}_{i \mid i-1}$ and $\hat{\nu}_{i \mid i-1}^{2}$ are given by Eq. (17) and (18) respectively. However, since $y_{i}$ is not directly traded in the market, we need to imply it from observed market prices, such as stock prices, and this is done using the approach described next. Under the credit framework described in Section II, and denoting by $T_{i}$ the maturity of the debt $K_{i}$, we have that the equity price at time $t_{i}$ may be expressed as

$$
\begin{aligned}
E\left(t_{i}, T_{i}\right) & =f\left(\hat{x}_{i \mid i}, \hat{\sigma}_{i \mid i}^{2}, K_{i}, r, \sigma\right) \\
& =g\left(y_{i}, \hat{x}_{i \mid i-1}, \hat{\sigma}_{i \mid i-1}^{2}, K_{i}, \mu, \sigma, h, \nu, r\right)
\end{aligned}
$$

where $f$ and $g$ are two deterministic functions. The first equality is obtained directly from Eq. (12). The last equality follows from decomposing $\hat{x}_{i \mid i}$ and $\hat{\sigma}_{i \mid i}^{2}$ using the updating step of the Kalman filter given in Eq. (20) and Eq. (21).

Expressing the equity at time $t_{i}$ in terms of $y_{i}$ is convenient because it would allow to imply $y_{i}$ through inversion of the function $g$, in case $g$ is invertible. This turns out to be the case since the first order derivative of $g$ with respect to $y_{i}$ is given by

$$
e^{\hat{x}_{i \mid i}+0.5 \hat{\sigma}_{i \mid i}^{2} N}\left(d_{1}\left(\hat{x}_{i \mid i}, K_{i}, \tau_{i}, \hat{\sigma}_{T_{i} \mid t_{i}}\right)-\frac{\hat{\sigma}_{i \mid i}}{\hat{\sigma}_{T_{i} \mid t_{i}}}\right) G_{i}
$$

where $\tau_{i}=T_{i}-t_{i}$. It is straightforward to check that the expression in Eq. (27) is positive. This allows to recursively

\begin{tabular}{|l||l|}
\hline Predictors & $\beta_{F}$ \\
\hline \hline Return on Asset (ROA) & $1.72(2.46)$ \\
\hline Growth in External Financing (EFG) & $3.64(4.89)$ \\
\hline Research and Development (R\&D) & $5.28(3.05)$ \\
\hline Investing cash flow (ICF) & $1.42(1.71)$ \\
\hline Outsider ownership & $1.21(1.03)$ \\
\hline (Outsider ownership) $^{2}$ & $1.45(0.76)$ \\
\hline Board size & $-0.02(-0.35)$ \\
\hline Log asset value (Log-Ass) & $0.09(0.65)$ \\
\hline Age & $0.01(1.78)$ \\
\hline Technology & $0.09(0.21)$ \\
\hline Service & $-0.32(-0.52)$ \\
\hline Trade & $0.88(-1.28)$ \\
\hline
\end{tabular}

TABLE II

MODEL PREDICTORS FOR THE POTENTIAL OF BIAS REPORTING 
imply the observations $y_{i}$ from the market prices of equities as described next. Let $e_{1}, \ldots, e_{i}$ be the market prices of equities, with $e_{j}$ denoting the equity price at time $t_{j}$. We define the following four-step procedure to imply the reported asset values $y_{j}$ from the equity price $e_{j}$.

(1) Predict the mean and variance of the true log-asset value using the time propagation formulas of the Kalman filter given in Eq. (15) and Eq. (16).

(2) Calculate the mean and variance of the predicted observed log-asset value using Eq. (17) and Eq. (18).

(3) Imply $y_{i}$ from $g$, i.e. numerically compute

$$
y_{i}=g^{-1}\left(e_{i} ; \hat{x}_{i \mid i-1}, \hat{\sigma}_{i \mid i-1}^{2}, K_{i}, \mu, \sigma, h(i), \nu, r\right)
$$

where the notation above indicates that we are considering $g$ as a function of the only variable $y_{i}$ and keeping the remaining parameters fixed.

(4) Update the mean and variance of the true log-asset value using the correction formulas of the Kalman filter given in Eq. (20) and Eq. (21).

Let $\boldsymbol{p}_{i}=\left(\hat{x}_{i \mid i-1}, \hat{\sigma}_{i \mid i-1}^{2}, K_{i}, \mu, \sigma, h(i), \nu\right)$ and denote $v_{i}=$ $g^{-1}\left(e_{i} ; \boldsymbol{p}_{i}\right)$. Then we can rewrite the log-likelihood function in terms of the observed market stock prices $e_{i}$. It may be proven that

$$
L L\left(e_{1}, e_{2}, \ldots, e_{n}\right)=L L\left(v_{1}, v_{2}, \ldots, v_{n}\right)-\sum_{i=1}^{n} \log \left(g^{\prime}\left(v_{i}\right)\right)
$$

where $g^{\prime}$ is the first order derivative of $g$ with respect to $y_{i}$ evaluated at $y_{i}=v_{i}$. The explicit expression corresponding to Eq. (29) is given by

$$
\begin{aligned}
& L L\left(e_{1}, e_{2}, \ldots, e_{n}\right)= \\
& =-\frac{n}{2} \log (2 \pi)-\sum_{i=1}^{n} \log \left(\hat{\nu}_{i \mid i-1}\right) \\
& -\sum_{i=1}^{n} \frac{\left(g^{-1}\left(e_{i} ; \boldsymbol{p}_{i}\right)-\hat{y}_{i \mid i-1}\right)^{2}}{2 \hat{\nu}_{i \mid i-1}^{2}} \\
& -\sum_{i=1}^{n} \log \left(G_{i}\right) \\
& -\sum_{i=1}^{n} \log \left(N\left(d_{1}\left(\hat{x}_{i \mid i}, K_{i}, \tau_{i}, \hat{\sigma}_{T_{i} \mid t_{i}}\right)-\frac{\hat{\sigma}_{i \mid i}}{\hat{\sigma}_{T_{i} \mid t_{i}}}\right)\right) \\
& -\sum_{i=1}^{n}\left(\hat{x}_{i \mid i}+0.5 \hat{\sigma}_{i \mid i}^{2}\right)
\end{aligned}
$$

where $\hat{y}_{i \mid i-1}$ is computed using Eq. (17) and $\hat{\nu}_{i \mid i-1}$ is computed using Eq. (18) in the step (2) of the above procedure. The value $y_{i}=g^{-1}\left(e_{i} ; \boldsymbol{p}_{i}\right)$ is implied from step (3), while $\hat{x}_{i \mid i}$ and $\hat{\sigma}_{i \mid i}^{2}$ are computed in step (4) along with $d_{1}$. The procedure given above reduces to Duan estimation algorithm in case of perfect observations. This is shown in Appendix III.

\section{Applications}

We apply the calibration methodology described in Section III to Tyco, a well known case of corporate reporting bias occurred in the United States history. Management misconduct was first discovered on March 13, 2001, when Tyco announced a 9.2 billion cash and stock deal to take over the CIT group, a commercial finance company. Tyco shares dropped sharply at the end of January 2002, as a proxy report was filed with the Securities and Exchange Commission disclosing that Tyco CFO got a 10 million dollars fee on the CIT Group deal, and that another 10 million went to a charity where he was a director. Figure 1 reports the CDS quotes for Tyco in the years 2001-2002. The crisis of end January 2002 appears very clearly, when the level of the CDS spread for the 1 year maturity doubled. Furthermore, the term structure of the CDS spreads changed to an inverted shape, so that the 3 year CDS was quoting on average about $100 \mathrm{bp}$ less than the 1 year contract, and the 5 year contract was quoting about $190 \mathrm{bp}$ lower. An inverted structure of the CDS spread is indeed typical of periods of particular stress. This means that the market prices a very high probability of default in the first year, while the probability is lower for the years further in the future. This is confirmed from the data of the cumulative default probability, bootstrapped from CDS data using standard procedures [1] and portrayed in Figure 2.

We can see from Figure 3 that a significant probability that reporting is biased gets estimated by the model during this period. Such probabilities $P_{m}(k)$ are calculated using Eq. (24) after computing the values of the predictors at a quarterly frequency, corresponding to times when balance sheet data are released. Such balance sheet indicators are obtained from Compustat. The resulting graph of the probability of biased reporting is presented in Figure 3.

We use the estimated values of $P_{m}(k)$ and make the assumption that

$$
h(k)=P_{m}(k) h
$$

i.e. we assume that the expected conditional amount of reporting bias $E[m(k) \mid\{m(k)>0\}]$ is constant throughout the period, with the only variability given by the probability $P_{m}(k)$ that the reporting is biased. We then calibrate the remaining parameters $(h, \mu, \sigma, \nu)$ using the estimation procedure illustrated in Section III-C. At each time $t_{i}$ we set the debt maturity $T_{i}=t_{i}+5$, i.e. assume that the debt is due five years from now. Moreover, we proxy the amount $K_{i}$ of debt due with the long term debt recovered from balance sheet data. We also run the Duan estimation procedure assuming a classical Merton model of credit risk. The obtained estimates are reported in Table III. The results

\begin{tabular}{|c|c||c|}
\hline & \multicolumn{2}{|c|}{ MLE Estimates } \\
\hline Parameters & Proposed Model & Merton Model \\
\hline \hline$\mu$ & $-7 \%(0.013)$ & $-8 \%(0.004)$ \\
\hline$\sigma$ & $23.2 \%(0.0022) \%$ & $37 \%(0.007)$ \\
\hline$h$ & $11 \%(0.0034)$ & \\
\hline$\nu$ & $1.2 \%(0.0012) \%$ & \\
\hline
\end{tabular}

TABLE III

PARAMETER ESTIMATES USING STOCK PRICE DATA FROM TYCO 
show that the expected reporting bias plays an important role in the specification of the model. If it were omitted, as it happens for the Merton model, then the reduced value of the firm's asset value due to reporting bias would simply be explained by an increase of the asset volatility which rises to $37 \%$. This exceeds typical volatility values found for firms in the same rating category which range in the interval $10 \%-30 \%$.

We use the estimates reported in Table III to predict the values of equity from time $t_{j}$ to time $t_{i}$ given the available information at time $t_{j}$ and using our calibrated credit risk model. The predicted value $\hat{e}_{i \mid j}$ of equity from $t_{j}$ to $t_{i}$ is calculated as

$$
\begin{aligned}
\hat{e}_{i \mid j} & =\mathbb{E}\left[E\left(t_{i}, T_{i}\right) \mid Y^{j}\right] \\
& =e^{-r\left(T_{i}-t_{i}\right)} \mathbb{E}\left[\mathbb{E}\left[\left(V_{T_{i}}-K_{i}\right)^{+} \mid Y^{i}\right] \mid Y^{j}\right] \\
& =e^{-r\left(T_{i}-t_{i}\right)} \mathbb{E}\left[\left(V_{T_{i}}-K_{i}\right)^{+} \mid Y^{j}\right] \\
& =e^{-5 r} \mathbb{E}\left[\left(V_{T_{i}}-K_{i}\right)^{+} \mid Y^{j}\right]
\end{aligned}
$$

where the second equality follows from the equity price given by Eq. (5), the third equality follows from the law of iterated expectations, and the last equality follows from the fact that the debt is assumed to have a five-year maturity. The last expectation is computed using Eq. (12) and the parameters in Table III returned from the calibration procedure. The prediction results are reported in table IV. The default threshold $K$ is assumed to be the same throughout the calibration period and proxied with the long term debt reported in the balance sheet statement released in the first quarter of 2001. The prediction is done one week ahead, for example the prediction for $1 / 16 / 2001$ is done on $1 / 8 / 2001$. We report prediction results in the period corresponding to the first three months of 2001, a period which was later found to be contaminated by managerial reporting bias.

\begin{tabular}{|l||l|l|l|}
\hline Dates & Market & Predicted & Merton \\
\hline \hline $1 / 8 / 2001$ & 53.96 & $54.65(1 \%)$ & $61.59(14 \%)$ \\
\hline $1 / 16 / 2001$ & 55.61 & $55.9(0.5 \%)$ & $63.21(13 \%)$ \\
\hline $1 / 22 / 2001$ & 60.7 & $60.13(-1 \%)$ & $68.2(12 \%)$ \\
\hline $1 / 29 / 2001$ & 61.72 & $61.3(-0.5 \%)$ & $69.3(12 \%)$ \\
\hline $2 / 5 / 2001$ & 63.37 & $62.71(-1 \%)$ & $70.86(12 \%)$ \\
\hline $2 / 12 / 2001$ & 62.49 & $62.15(-0.5 \%)$ & $70.03(12 \%)$ \\
\hline $2 / 20 / 2001$ & 60.78 & $60.68(0.1 \%)$ & $68.28(12 \%)$ \\
\hline $2 / 26 / 2001$ & 58.64 & $58.78(0.2 \%)$ & $66.12(13 \%)$ \\
\hline $3 / 5 / 2001$ & 56.28 & $56.82(1 \%)$ & $63.87(13 \%)$ \\
\hline $3 / 12 / 2001$ & 53.95 & $54.74(1 \%)$ & $61.53(14 \%)$ \\
\hline $3 / 19 / 2001$ & 51.62 & $52.7(2 \%)$ & $59.23(15 \%)$ \\
\hline $3 / 26 / 2001$ & 46.22 & $48.1(4 \%)$ & $53.83(16 \%)$ \\
\hline $4 / 2 / 2001$ & 45.35 & $47.0(3 \%)$ & $52.96(16 \%)$ \\
\hline
\end{tabular}

TABLE IV

THE TABLE SHOWS THE ACTUAL MARKET PRICE OF TYCO STOCK, THE STOCK PRICE PREDICTED BY OUR MODEL, AND THE STOCK PRICE PREDicted USING MERTON MODEL. THE VALUeS IN PARENTHESIS INDICATE THE ERROR COMMITTED BY EACH METHOD.

Table IV shows that the predictions obtained using our calibrated credit risk framework are very accurate and exhibit an average error of $1 \%$. The prediction obtained using Merton model instead is more inaccurate and at times exceeds $16 \%$.
The superior performance of our method may be explained as follows. Equity price in Merton model is a call option on the firm's asset value, as seen from Eq. (5). The option price increases as the asset value of the firm increases. However, our calibration results in Table III show a non-negligible amount of reporting bias which reduces the actual asset value of the firm, and consequently reduces the equity price. By contrast, Merton model does not adjust for reporting bias and prices equity assuming a larger initial firm's asset value, thus overestimating the market stock price of equity. Therefore, the results confirm that in cases of occurred misreporting, the explicit modeling of reporting bias leads to downward corrections of the stock price predicted by the Merton model which match more accurately the market stock price.

\section{CONCLUSIONS}

We have presented a calibration scheme for the credit model with reporting bias presented in [3]. The calibration method consists of two stages. In the first stage the probability that the manager introduces bias at a specific time $t$ is calculated using balance sheet data and financial indicators. In the second stage, the obtained estimate is then used along with a transformed maximum likelihood estimation procedure to estimate the remaining model parameters, namely the drift and asset volatility, the expected amount of reporting bias, and the noisy variance in the reported asset value. We validated the proposed methodology on Tyco, a real case of occurred reporting bias, and showed that the calibrated model has a significant power in predicting the market price of equity one week ahead.

\section{REFERENCES}

[1] D. Brigo, F. Mercurio. Interest Rate Models: Theory and Practice, Springer Finance, 2nd edition, 2006.

[2] D. Brigo, M. Morini. "CDS Calibration with tractable structural models under uncertain credit quality". Risk Magazine 19, 2006.

[3] A. Capponi, J. Cvitanic. "Credit Risk Modeling with Misreporting and Incomplete Information". To appear in International Journal of Theoretical and Applied Finance 12, 2009.

[4] U. Cetin, R. Jarrow, P. Protter, Y. Yildirim. "Modeling credit risk with partial information". Annals of Applied Probability, 14, 1167-1178, 2004.

[5] D. Coculescu, H. Geman, M. Jeanblanc. "Valuation of Default Sensitive Claims Under Imperfect Information", Working paper, 2006.

[6] J. Duan. "Maximum Likelihood Estimation Using Price Data of the Derivative Contract". Mathematical Finance, 4, 155-167, 1994.

[7] J. Duan. "Correction: Maximum Likelihood Estimation Using Price Data of the Derivative Contract". Mathematical Finance, 10, 461-462, 2000.

[8] D. Duffie, D. Lando. "Term structure of credit spreads with incomplete accounting information". Econometrica, 63, 633-664, 2001

[9] X. Guo, R.A. Jarrow, Y. Zeng. "Credit risk models with incomplete information". Forthcoming in Mathematics of Operations Research, 2008.

[10] R. E. Kalman. "A new approach to linear filtering and prediction problems". Transactions of the ASME - Journal of Basic Engineering on Automatic Control, 82, 35-45, 1960.

[11] R. C. Merton. "On the pricing of corporate debt: the risk structure of interest rates", Journal of Finance 29, 449-470, 1974.

[12] F. Yu. "Accounting transparency and the term structure of credit spreads". Journal of Financial Economics, 75, 53-84, 2005.

[13] T. Wang. "Real Investment and Corporate Securities Fraud". Working paper, University of Minnesota, 2007. 
APPENDIX I

SUM OF BOND AND EQUITY PRICE

Tyco CDS

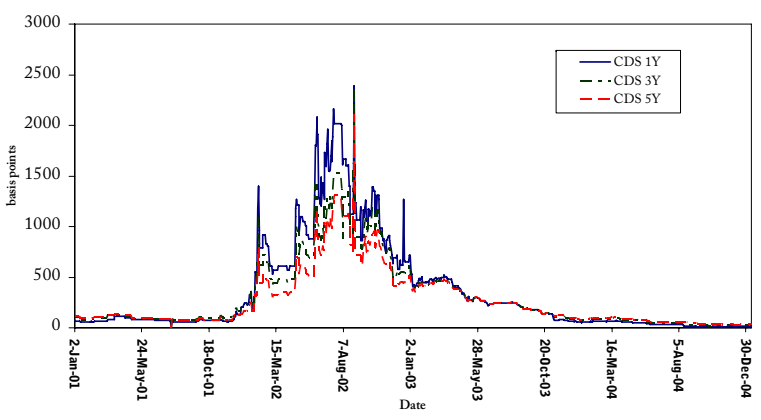

Fig. 1. The term structure of CDS of Tyco

Tyco Default Probability

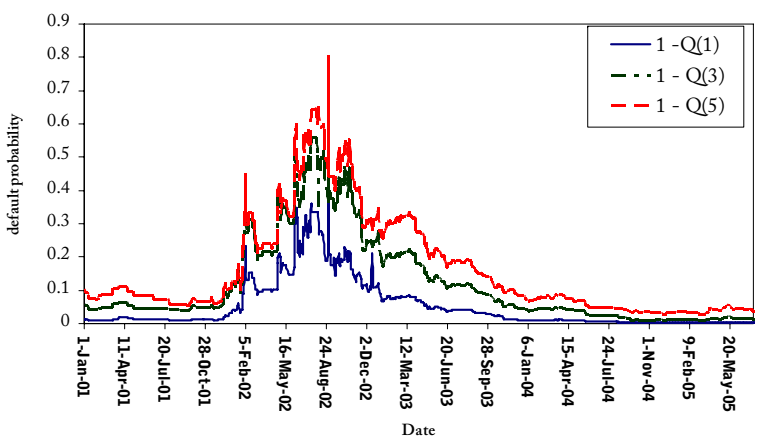

Fig. 2. Default Probability of Tyco

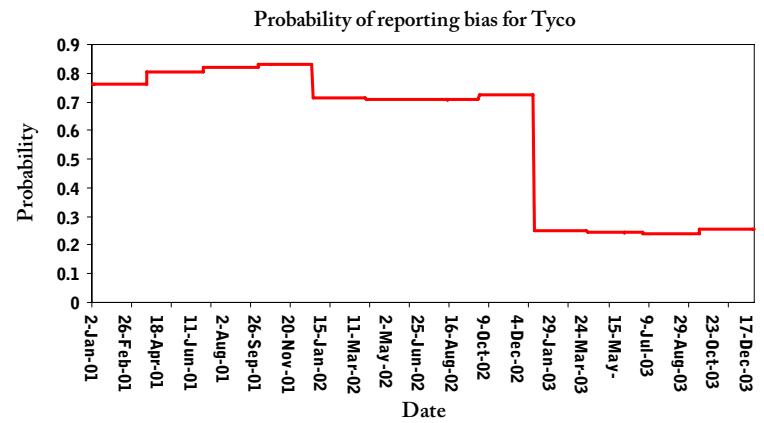

Fig. 3. The probability of biased reporting for Tyco
We prove that

$$
\mathbb{E}\left[V_{t} \mid Y^{t}\right]=E(t, T)+B(t, T)
$$

in our credit risk framework, under the measure $P^{*}$ obtained replacing $\mu$ with the risk free interest rate $r$. We first notice that, regardless of whether the event $\left\{K \geq V_{T}\right\}$ happens, we have

$$
E(t, T)+B(t, T)=e^{-r \tau} \mathbb{E}\left[V_{T} \mid Y^{t}\right]
$$

The result follows from

$$
\begin{aligned}
\mathbb{E}\left[V_{T} \mid Y^{t}\right] & =E\left[V_{t} e^{\left(r-\frac{1}{2} \sigma^{2}\right) \tau+\sigma\left(W_{T}-W_{t}\right)} \mid Y^{t}\right] \\
& =e^{r \tau} E\left[V_{t} \mid Y^{t}\right]
\end{aligned}
$$

\section{APPENDIX II}

\section{NO OUTSIDER ARBITRAGE}

We want to prove that the discounted price process $M(t)=E\left[V_{t} \mid Y^{t}\right]$ is a martingale. Let $s<t$. We have

$$
\begin{aligned}
& e^{-r(t-s)} E\left[M(t) \mid Y^{s}\right] \\
& \quad=e^{-r(t-s)} E\left[E\left[V_{t} \mid Y^{t}\right] \mid Y^{s}\right] \\
& \quad=e^{-r(t-s)} E\left[V_{t} \mid Y^{s}\right] \\
& \quad=e^{-r(t-s)} E\left[V_{s} e^{\left(r-\frac{1}{2} \sigma^{2}\right)(t-s)+\sigma\left(W_{t}-W_{s}\right)} \mid Y^{s}\right] \\
& \quad=M_{s}
\end{aligned}
$$

\section{APPENDIX III}

\section{REDUCTION TO DUAN'S APPROACH}

Under the assumption that reporting is not biased $(h(k)=$ $0, \nu=0)$, the observation equation in the system given by Eq. (13) would reduce to $y_{k}=x_{k}$, and we would have that

$$
E(t, T)=C\left(t, V_{t}, K, \sigma, T\right)
$$

which $C\left(t, V_{t}, K, \sigma, T\right)$ denotes the time $t$ price of a call option on the asset value with strike $K$, initial asset price $V_{t}$, volatility $\sigma$ and maturity $T$. Moreover, we have

$$
\begin{aligned}
d_{1}\left(\hat{x}_{i \mid i}, K_{i}, \tau_{i}, \hat{\sigma}_{T_{i} \mid t_{i}}\right) & =\frac{x_{i}-\log \left(K_{i}\right)+\left(r+0.5 \sigma^{2}\right) \tau_{i}}{\sigma \sqrt{\tau_{i}}} \\
& :=d_{1}\left(x_{i}, K_{i}, \tau_{i}\right) \\
G_{i} & =1 \forall i \\
\hat{\nu}_{i \mid i-1}^{2} & =\sigma^{2} \Delta_{i} \\
\hat{x}_{i \mid i} & =x_{i} \\
\hat{\sigma}_{i \mid i} & =0 \\
\hat{y}_{i \mid i-1} & =x_{i}+\left(\mu-0.5 \sigma^{2}\right) \Delta_{i}
\end{aligned}
$$


Using the limiting results given in Eq. (37), we would have that the log-likelihood function given in Eq. (30) reduces to

$$
\begin{aligned}
L L\left(e_{1}, e_{2}, \ldots, e_{n}\right)= \\
=-\frac{n}{2} \log (2 \pi)-n \log (\sigma)-\frac{1}{2} \sum_{i=1}^{n} \log \left(\Delta_{i}\right) \\
\quad-\frac{1}{2 \sigma^{2}} \sum_{i=1}^{n} \frac{\left(x_{i}(\sigma)-x_{i-1}(\sigma)-\left(\mu-0.5 \sigma^{2}\right) \Delta_{i}\right)^{2}}{\Delta_{i}} \\
\quad-\sum_{i=1}^{n} x_{i}(\sigma)-\sum_{i=1}^{n} \log \left(N\left(d_{1}\left(x_{i}(\sigma), K_{i}, \tau_{i}\right)\right)\right)(37)
\end{aligned}
$$

where $x_{k}(\sigma)=\log \left(C^{-1}\left(e_{k} ; \sigma\right)\right)$ is the true log-asset value implied by inversion of the call pricing formula for the equity price. Eq. (37) corresponds to the log-likelihood function in Duan's estimation procedure [6] [7]. 